\title{
A Quantitative Evaluation of Microstructure by Electron Back-Scattered Diffraction Pattern Quality Variations
}

\author{
Suk Hoon Kang, ${ }^{1}$ Hyung-Ha Jin, ${ }^{1}$ Jinsung Jang, ${ }^{1, \star}$ Yong Seok Choi, ${ }^{2}$ Kyu Hwan Oh, ${ }^{2}$ \\ David C. Foley, ${ }^{3}$ and Xinghang Zhang $^{3}$ \\ ${ }^{1}$ Nuclear Materials Division, Korea Atomic Energy Research Institute, Daejeon 305-353, Korea \\ ${ }^{2}$ Department of Materials Science and Engineering, Seoul National University, Seoul 151-744, Korea \\ ${ }^{3}$ Department of Mechanical Engineering, Texas A\&M University, College Station, TX 77843, USA
}

\begin{abstract}
Band contrast (BC) is a qualitative measure of electron back-scattered diffraction (EBSD), which is derived from the intensity of the Kikuchi bands. The $\mathrm{BC}$ is dependent upon several factors including scanning electron microscope measurement parameters, EBSD camera setup, and the specimen itself (lattice defect and grain orientation). In this study, the effective factors for $\mathrm{BC}$ variations and the feasibility of using $\mathrm{BC}$ variations for the quantification of microstructure evolutions have been investigated. In addition, the effects of the lattice defect and the grain orientation on the BC variations are studied. Next, a shear-deformed microstructure of 316L stainless steel, which contains nanosized grains and a large portion of twin boundaries, is revealed by BC map and histogram. Recovery and recrystallization of shear-deformed 316L stainless steel are displayed by BC variations during isothermal annealing at 700 and $800^{\circ} \mathrm{C}$, respectively. It is observed that the $\mathrm{BC}$ turns bright as the shear-deformed crystal structure is recovered or recrystallized.
\end{abstract}

Key words: EBSD, band contrast, annealing, microstructure, recovery, recrystallization

\section{INTRODUCTION}

$\mathrm{X}$-ray or neutron beam diffraction is a conventional tool for determining the variations of crystal lattice structures in a macroscale, and it make measurements on innumerable grains. Electron beam diffraction is a tool for determining such variations in a microscale, and it makes measurements on a grain-by-grain basis. Many important behaviors of the material are controlled by the local characteristics rather than by global averages, and this has been a strong driving force in the development of electron beam applications, especially the electron back-scattered diffraction (EBSD) method (Wilkinson \& Dingley, 1991, 1992; Krieger Lassen et al., 1992; Wilkinson et al., 1992; Mukherjee et al., 1995).

With the advanced spatial resolution, EBSD has been widely used to probe both elastic and plastic strains. Basically, unstrained materials show sharper and higher-quality EBSD patterns. However, the presence of elastic strain, or population of defects associated with the strain, leads to the broadening of diffracted patterns and lower quality of EBSD pattern. For example, the distribution of plastic deformation in $\mathrm{Al}$ matrix composites reinforced with $\mathrm{SiC}$ fibres are characterized by EBSD pattern variations (Wilkinson \& Dingley, 1992; Wilkinson et al., 1992; Mukherjee et al., 1995). The quality of the EBSD pattern is usually quantified by band contrast (BC), which is derived from the average intensity of the Kikuchi bands with respect to the overall intensity within an EBSD pattern. BC can be evaluated by $256(0-255)$ different levels of brightness: "0" corresponds to the bad EBSD pattern quality and black color, and " 255 " corresponds to the good EBSD pattern quality and white color. The ob-

() MICROSCOPY SOCIETY OF AMERICA 2013

${ }^{\star}$ Corresponding author. E-mail: jjang@kaeri.re.kr served brightness can be mapped into a grayscale image, and it shows detailed features of the microstructure, such as boundaries. The statistical distribution of brightness can be plotted in a diagram, and it can be quantitatively compared with diagrams of other samples (BC histogram).

The EBSD pattern is generally obtained by $70^{\circ}$ tilting of a sample under a scanning electron microscope (SEM). The interaction depth between the incident electron beam and the sample is very shallow (tens of nanometers), and most of the diffracted signal is generated from the sample surface. Therefore, the signals are very sensitive to the SEM/EBSD measurement condition and surface topology. Before evaluating material characteristics, it should be confirmed that the EBSD pattern and the corresponding $\mathrm{BC}$ are always consistent with each other regardless of the measurement condition or surface topology.

In this study, the effective parameters for $\mathrm{BC}$ variations are investigated, and the feasibility of using $\mathrm{BC}$ variations for quantification of microstructure evolutions has been studied. The BC variation factors such as SEM parameters, EBSD camera setup, lattice defects, and grain orientations were investigated, and the recovery and recrystallization of the sample were quantified by BC map and histogram.

\section{Materials and Methods}

Four groups of samples were used in this study: dual-phase steel ( $\mathrm{Fe}-1.3 \mathrm{Mn}-0.3 \mathrm{C})$, interstitial-free (IF) steel, gold wire, and simple shear-deformed type 316L stainless steel. Dualphase steel $(\mathrm{Fe}-1.3 \mathrm{Mn}-0.3 \mathrm{C})$ was used to investigate the relationship between the SEM/EBSD parameters and BC variations. Two phases, ferrite phase with a body-centered cubic (bcc) structure and martensite phase with a BCT 

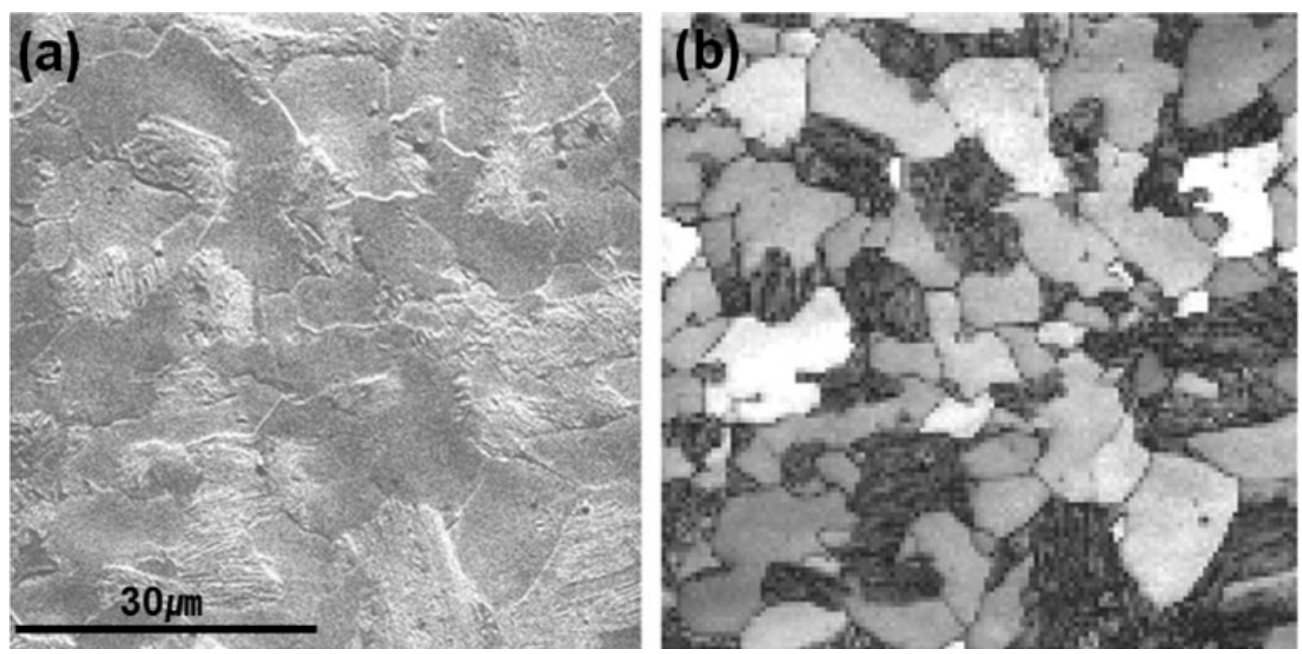

Figure 1. a: Scanning electron microscope micrograph and (b) electron back-scattered diffraction band contrast of $\mathrm{Fe}-1.3 \mathrm{Mn}-0.3 \mathrm{C}$ dual-phase steel. Martensite phase is indicated as a dark-colored one, and the ferrite phase is indicated as a bright-colored one.

structure, showed two characteristically different peaks in the $\mathrm{BC}$ histogram, and they appeared useful to evaluate the effects of the SEM measuring parameters and EBSD camera setup on the $\mathrm{BC}$ variations.

An IF steel sample was used to investigate the effect of grain orientations on the $\mathrm{BC}$ variations. Recrystallized grains of IF steel clearly showed the orientation differences of each grain, and the corresponding $\mathrm{BC}$ differences of each grain were evaluated.

A recrystallized gold wire sample was used to investigate the relationship between the lattice strain and $\mathrm{BC}$ distributions. The grain and grain boundaries could be distinguished by a particular value of $\mathrm{BC}$, and the corresponding grain map has been estimated.

Simple shear-deformed 316L stainless steel was used for the investigation of the relationship of the recovery and recrystallization with $\mathrm{BC}$ variations. The samples were subjected to an annealing process at recovery or recrystallization temperature, respectively. Square-shaped indent marks were made on the sample surface, and the same area was observed before and after the heat treatment.

To ensure that all the samples have the same surface condition, samples were subjected to electrolytic polishing under the same voltage and etchant flow condition. Electropolishing is effective in relieving the local defects on the surface, which is usually generated during mechanical polishing of samples. An SEM/EBSD system (JSM 6500F/Oxford Inca) was used to observe the microstructural evolution and the BC variations. An acceleration voltage of $20 \mathrm{kV}$ with $4 \mathrm{nA}$ probe current was used for SEM, and 100-ms integration time and 100 video gains for EBSD analyses.

\section{Results}

SEM Measurement Parameters, EBSD Setup, and BC An SEM micrograph and an EBSD BC map of Fe-1.3Mn$0.3 \mathrm{C}$ dual-phase steel are shown in Figure 1. Two different phases in a dual-phase steel sample are identified by different brightness levels of the EBSD signal. The martensite phase is a dark-colored one, and the ferrite phase is brightcolored one. As the crystal structure of a martensite phase and a ferrite phase are all based on the bcc, they can hardly be distinguished by indexing of individual diffraction patterns. However, the formation of martensite in a ferrite matrix causes a high local dislocation density and high residual stress; therefore, the EBSD quality of the martensite phase is much lower than that of the ferrite phase, and the corresponding $\mathrm{BC}$ is dark.

The different phases have different $\mathrm{BC}$ values in the histogram as shown in Figure 2. Three characteristic peaks are observed in Figure 2a. The BC value of the first peak is estimated as 75 , the second one as 150 , and the last one as 220 , respectively. The first peak is identified as a martensite, and the last two peaks are identified as ferrite. The second and the third characteristic peaks in Figure 2a differ in crystal orientation. The grain orientation dependency of BC in the bcc structure is discussed later (Fig. 3).

In Figures $2 \mathrm{~b}-2 \mathrm{~d}, \mathrm{BC}$ histograms are displayed with different combinations of SEM measurement parameters and EBSD camera setup. The positions of characteristic peaks from the different phases and the $\mathrm{BC}$ histogram width $(\triangle B C)$ appear to be changed with SEM/EBSD measurement parameters. The relationship of $\mathrm{BC}$ variation with the SEM/EBSD measurement conditions is summarized in Figure 2e. It is observed that $\triangle \mathrm{BC}$ is proportional to the product of all parameters investigated such as acceleration voltage, probe current, integration time, and video gain. Three factors, except the acceleration voltage, directly affect the amount of input signals, resulting in proportionally varying signals. It is observed that the $\triangle \mathrm{BC}$ decreased by half following the decrease of the probe current from 4 to $2 \mathrm{nA}$, the integration time from 100 to $50 \mathrm{~ms}$, and the video gain from 100 to 50, respectively. It is also estimated that the acceleration voltage variation from 20 to $15 \mathrm{kV}$ induced a 

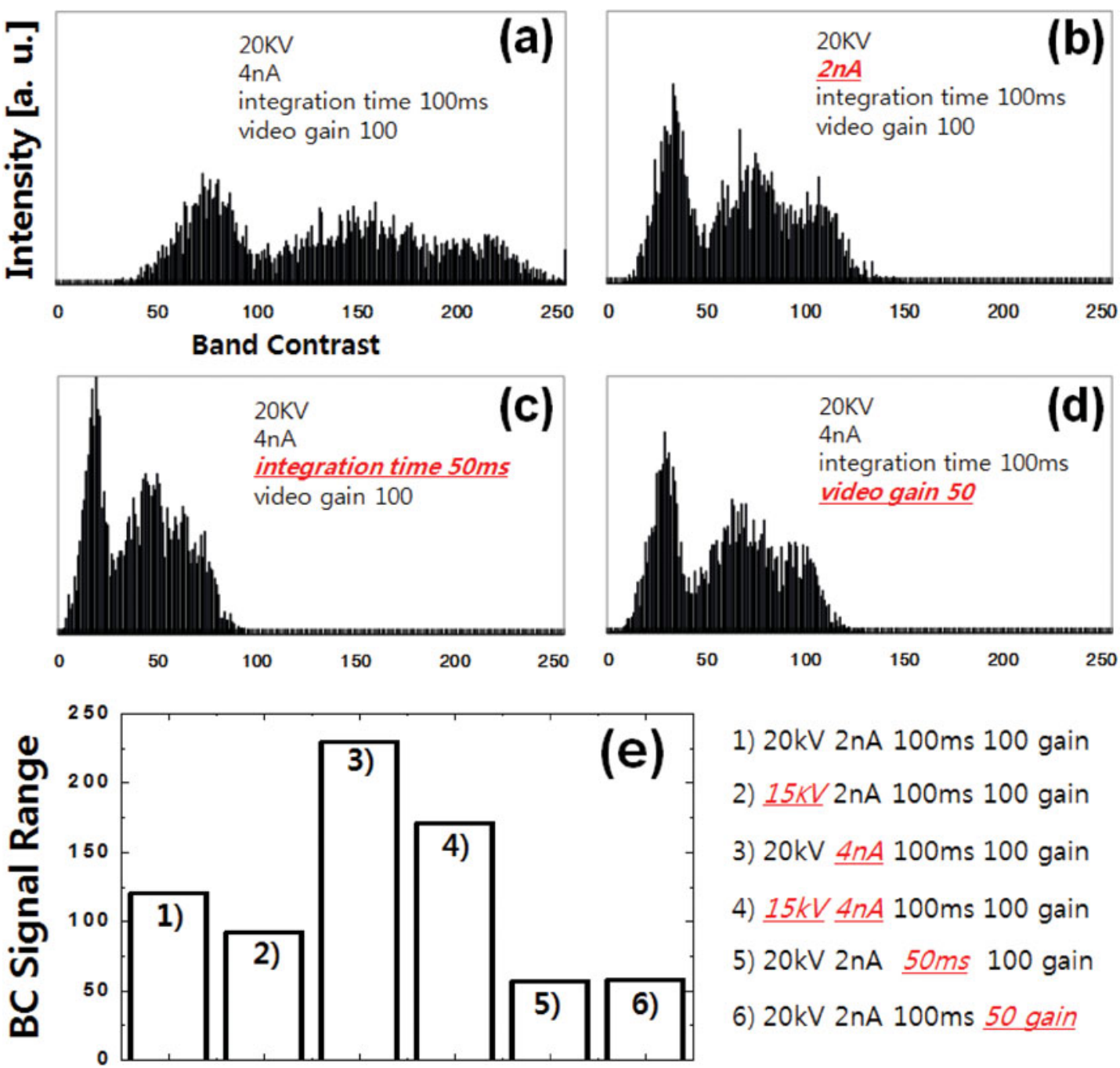

1) $20 \mathrm{kV} 2 \mathrm{nA} 100 \mathrm{~ms} 100$ gain

2) $15 \mathrm{KV} 2 \mathrm{nA} 100 \mathrm{~ms} 100$ gain

3) $20 \mathrm{kV} 4 n A 100 \mathrm{~ms} 100$ gain

4) $15 \mathrm{kV} 4 \mathrm{nA} 100 \mathrm{~ms} 100$ gain

5) $20 \mathrm{kV} 2 \mathrm{nA} 50 \mathrm{~ms} 100$ gain

6) $20 \mathrm{kV} 2 \mathrm{nA} 100 \mathrm{~ms} 50$ gain

Figure 2. Band-contrast histograms of a Fe-1.3Mn-0.3C dual-phase steel sample: (a) reference condition, (b) half probe current, (c) half integration time, and (d) half video gain. e: Summary of the relationship between band-contrast variations and scanning electron microscope/electron back-scattered diffraction measuring conditions.

decrease of $\triangle \mathrm{BC}$ by $\sim 0.75$ times-another proportional variation. Basically, $\mathrm{BC}$ quantifies the signals within the interaction volume between the electron beam and the specimen. Many researchers suggest that there is a linear relationship between the accelerating voltage and the interaction volume (Engler \& Randle, 2010). And it could be reasonable that acceleration voltage and $\mathrm{BC}$ variations shows a proportional relationship under the present experimental condition.

\section{Crystal Orientation and BC}

In Figure 3, the relationship between crystal orientation and $\mathrm{BC}$ value is illustrated. It is expected that some planes in the crystal lattice produce bands of higher intensity than others. A first-order approximation of the intensity of a diffraction band can be obtained by assuming the role of the structure factor. If the lattice is in an orientation such that the pattern contains many high-intensity reflections, then the pattern may have a higher BC value than the other cases (Wright \& Nowell, 2006). Thus, there will be some grain-to-grain variations in $\mathrm{BC}$ simply because of the grain orientation effect. Among fully recrystallized ferrite grains in IF steel in Figure $3 \mathrm{a}$, nine grains were identified by indexing of the corresponding patterns, and the $\mathrm{BC}$ map is displayed in Figure $3 \mathrm{~b}$. The different orientation of each grain is indexed in an inverse pole figure of plane normal direction in Figure 3c. From "grain 1" to "grain 9," the crystal orientation is indexed as (001), (013), (233), (111), (137), (113), (144), (011), and (011), respectively. In Figure 3d, BC values of each grain orientations are marked on the $\mathrm{BC}$ histogram. It is observed that the grains close to the (011) plane reveal the highest $\mathrm{BC}$ values. It is also true that (011) plane generates the highest peak in the X-ray diffraction of the bcc lattice.

\section{Lattice Strain and BC}

In Figure 4, three different areas of one gold wire sample are observed by EBSD. The BC values vary with different grain orientations, and the $\mathrm{BC}$ histogram shows different distributions of peaks because only several grains are included in 

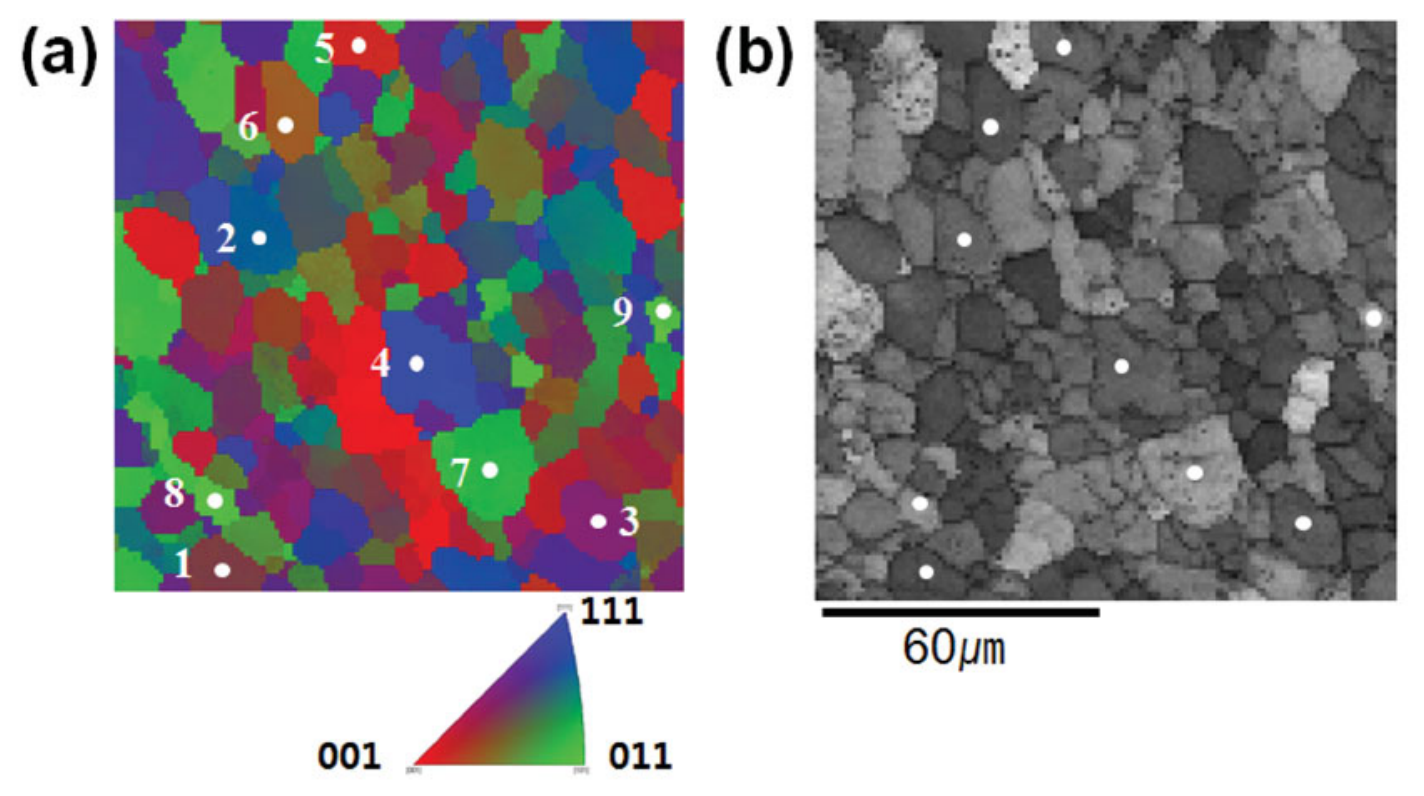

(c)

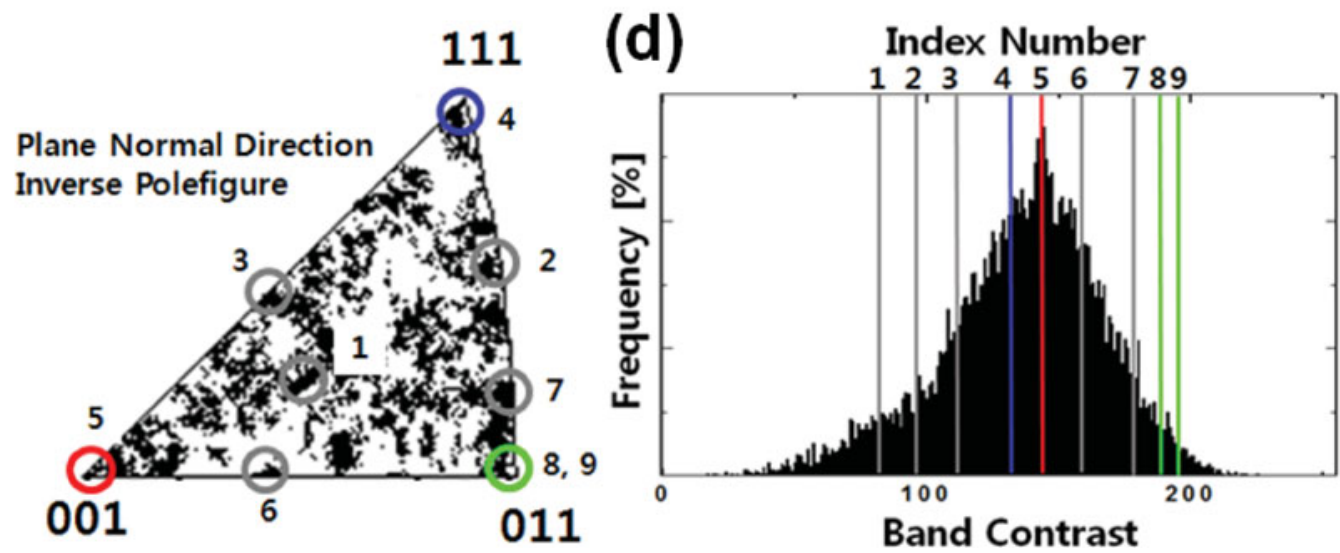

Figure 3. Crystallographic orientation dependency of band contrast are observed from an interstitial-free steel. a: Grains displayed by random brightness (grains are recrystallized, and there is no preferred orientation). Some grains that have specific orientation along the normal direction (ND) have been identified by numbers (b) corresponding to the grain map of band contrast; (c) numbered grains are expressed in the ND inverse pole space. d: Peak intensity and positions of numbered grains are indexed in the histogram of band contrast.

each map. It is found that a particular BC value could distinguish the grains from the grain boundaries in the histograms. In Figure 4d, an arbitrary number of 90 for a $\mathrm{BC}$ value is indicated; i.e., when the $\mathrm{BC}$ value is $>90$, the $\mathrm{BC}$ appears to be highly dependent on the crystal orientation of the sample; however, when the $\mathrm{BC}$ value is $\angle 90$, the $\mathrm{BC}$ is dependent on the lattice strain or defects in the sample such as dislocations and grain boundaries, not on the crystal orientation.

\section{Recovery, Recrystallization, and BC}

In Figure 5, microstructural evolution of a shear-deformed 316L stainless-steel sample after isothermal annealing is illustrated by $\mathrm{BC}$ variations. The same areas of the sample are observed before and after the annealing treatment. In Figures $5 \mathrm{a}$ and $5 c$, the shear band shows an inclination of about $45^{\circ}$ from the shear direction, but the grains appear parallel to the shear band direction. In Figures $5 \mathrm{~b}$ and $5 \mathrm{~d}$, the microstructural evolutions are shown by $\mathrm{BC}$ variations after isothermal annealing at 700 and $800^{\circ} \mathrm{C}$, respectively. The configuration of the $\mathrm{BC}$ map including the fine twin boundaries does not seem to have changed much after the annealing process at $700^{\circ} \mathrm{C}$, and the micrometer-sized grains appear similar in Figures $5 \mathrm{a}$ and $5 \mathrm{~b}$. When the annealing temperature is raised to $800^{\circ} \mathrm{C}$, it is observed that grains are mostly recrystallized. In Figure 5d, uniform equi-axed grains are developed by recrystallization, and the $\mathrm{BC}$ map becomes clearer. It is generally accepted that twin boundaries are highly resistant to boundary deterioration during annealing, and the dark area looks relatively stable because of the high boundary density of twin boundaries. The $\mathrm{BC}$ histograms in Figure 6, which correspond to pre- and postannealing processes of the shear-deformed 316L stainless steel specimens, suggest that the position of the peak moved to the higher $\mathrm{BC}$ value after annealing. The brightness change seems to be related to the grain boundary density. In Fig- 


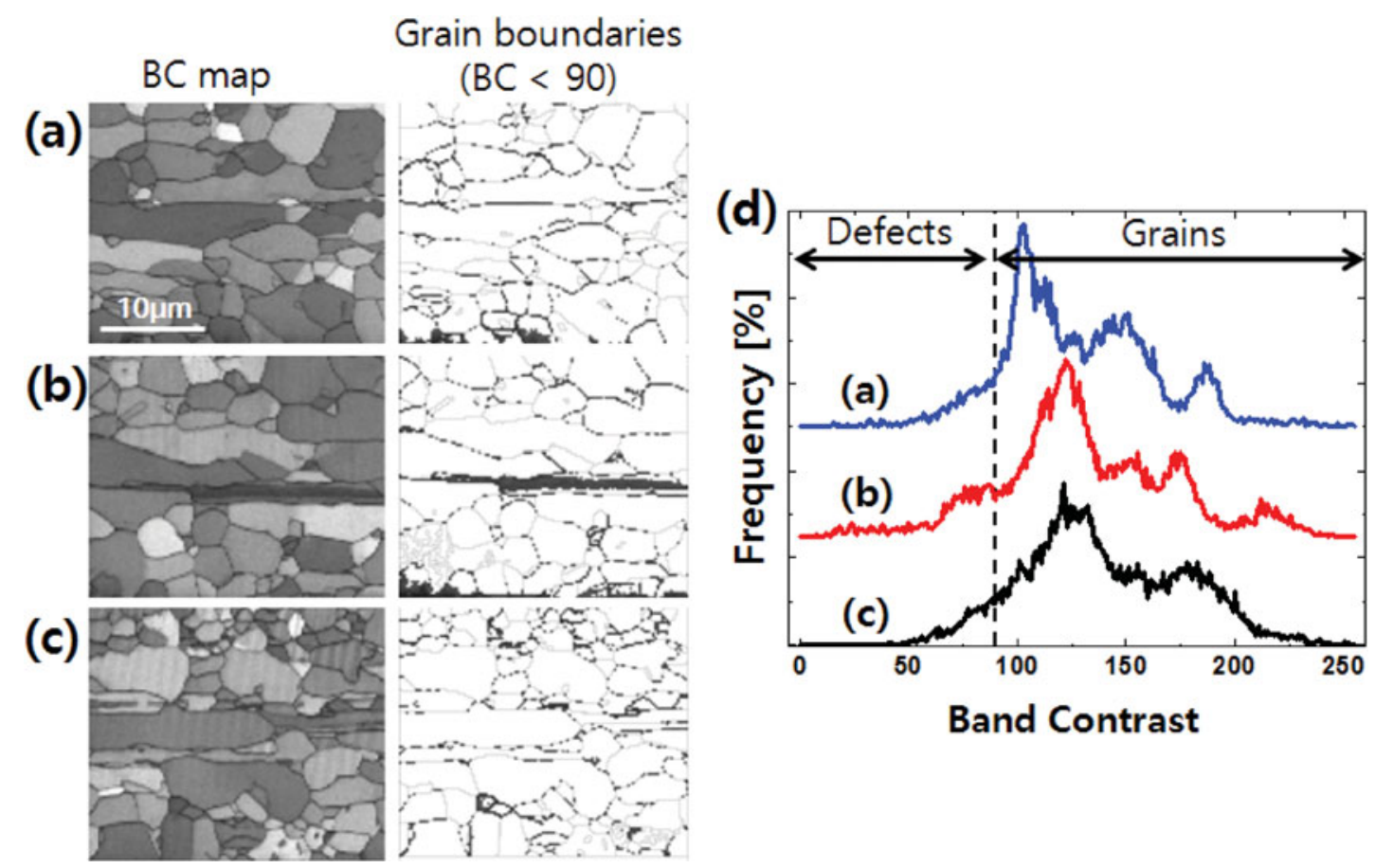

Figure 4. Band contrast (BC) maps of a recrystallized $99.9999 \%$ pure gold wire sample; a specific BC value of 90 is indicated for estimating three different $\mathrm{BC}$ histograms.
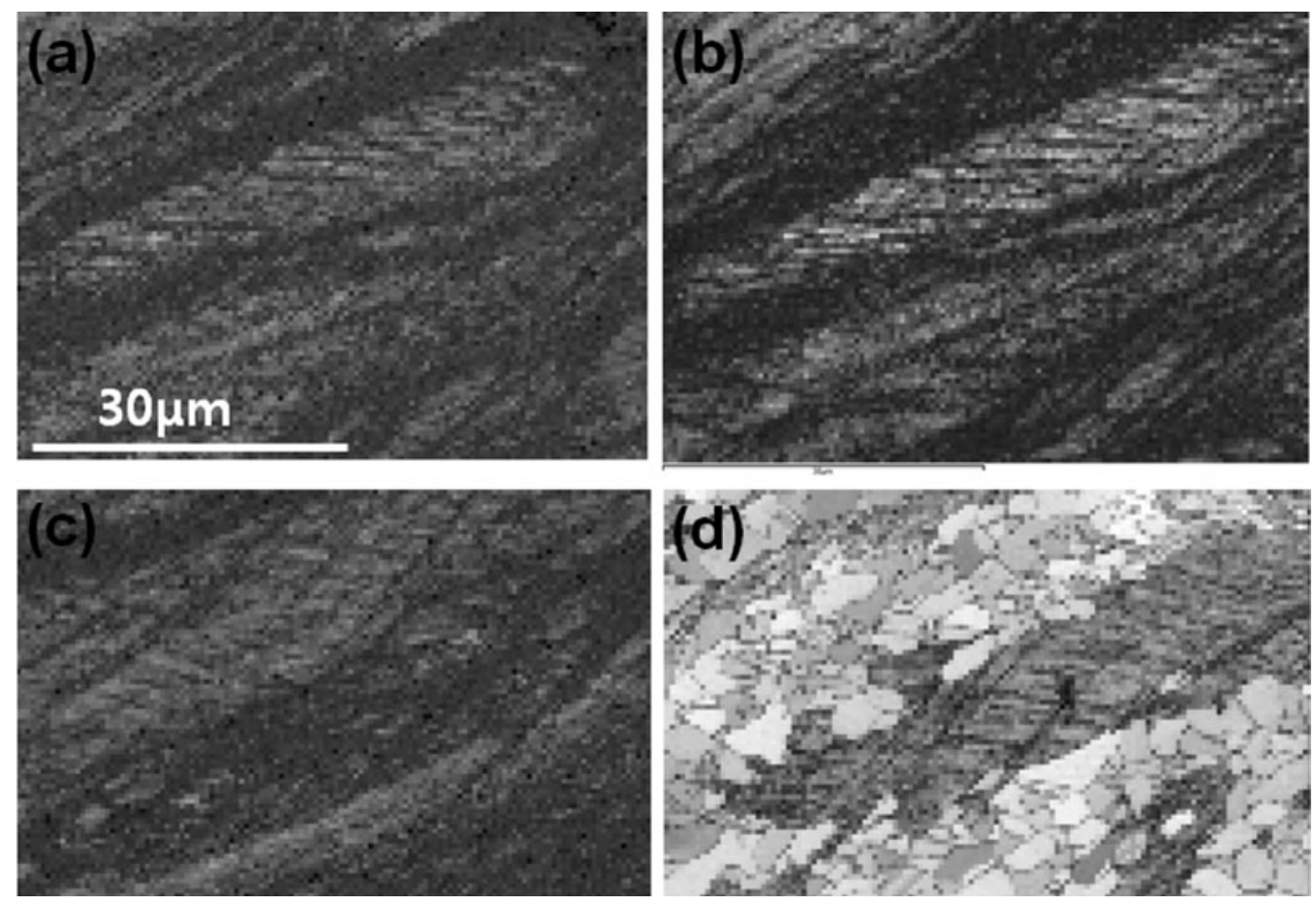

Figure 5. Band contrast (BC) maps of a shear-deformed 316L stainless steel sample. a, c: BC map of an ECAP-processed sample before the annealing process, and BC map the sample after annealing (b) at $700^{\circ} \mathrm{C}$ and (d) $800^{\circ} \mathrm{C}$ for $1 \mathrm{~h}$.

ure 6a, two peaks of high boundary density and low boundary density areas are closely positioned with not much difference in $\mathrm{BC}$ values. After recrystallization in Figure $6 \mathrm{~b}$, however, two peaks are separated into two distant ones; in other words, two peaks of high and low boundary density areas are readily distinguishable by $\mathrm{BC}$ values.

\section{SUMMARY}

In this study, the effective parameters for $\mathrm{BC}$ variations are investigated and the feasibility of using $\mathrm{BC}$ variations for quantification of microstructure evolutions has been studied. It is found that the $\mathrm{BC}$ is in proportion to SEM 

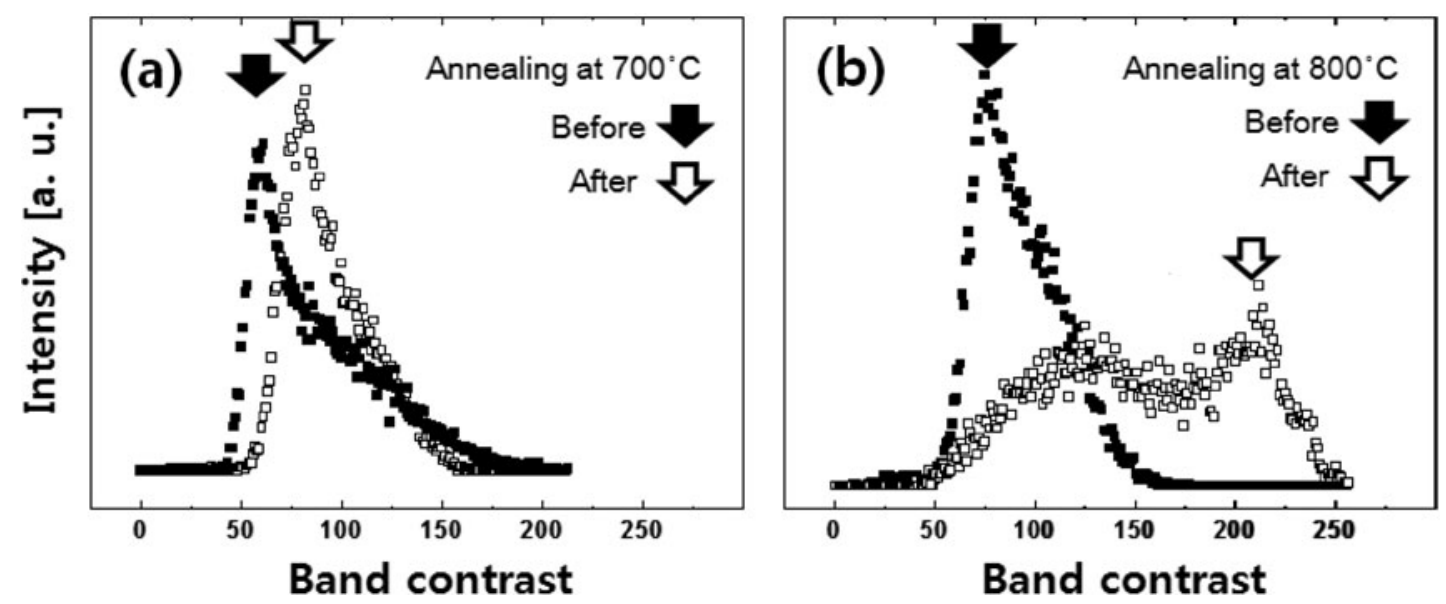

Figure 6. Band contrast (BC) histogram variations of shear-deformed 316L stainless steel samples before and after the annealing at (a) $700^{\circ} \mathrm{C}$ and (b) $800^{\circ} \mathrm{C}$ for $1 \mathrm{~h}$. The intensity of the brighter region became lowered and the position of the peak moved to the higher $\mathrm{BC}$ value.

measurement parameters of acceleration voltage and probe current and the EBSD camera setup of integration time and video gain. The relationship between $\mathrm{BC}$ and crystal orientation is investigated, and it is found that the grain that has closer orientation to the (011) plane shows the highest $\mathrm{BC}$ value. A particular $\mathrm{BC}$ value of 90 which distinguishes the grain boundaries from grains is proposed in this study. The microstructure of shear-deformed 316L stainless steel is characterized by the BC map and histogram. It is observed that the $\mathrm{BC}$ becomes brighter as the crystal structure of the shear-deformed $316 \mathrm{~L}$ stainless steel is recovered by an annealing at $700^{\circ} \mathrm{C}$, and becomes clearer and distinguishable by $\mathrm{BC}$ value when recrystallized by annealing at $800^{\circ} \mathrm{C}$.

\section{ACKNOWLEDGMENT}

This research was supported by Nuclear R\&D program through the National Research Foundation of Korea (NRF) funded by the Ministry of Education, Science and Technology (2011-0094107).

\section{ReFERENCES}

Engler, O. \& Randle, V. (2010). Introduction to Texture Analysis. Boca Raton, London, New York: CRC Press.

Krieger Lassen, N.C., Juul Jensen, D. \& Conradsen, K. (1992). Image-processing procedures for analysis of electron back scattering patterns. Scanning Microsc 6, 115-121.

Mukherjee, S., Garmestani, H. \& Chandra, N. (1995). Experimental investigation of thermally-induced plastic-deformation in MMCs using back scattered Kikuchi method. Scr Metall Mater 33, 93-99.

Wilkinson, A.J. \& Dingley, D.J. (1991). Quantitative deformation studies using electron back scatter patterns. Acta Metall Mater 39, 3047-3055.

Wilkinson, A.J. \& Dingley, D.J. (1992). The distribution of plastic deformation in a metal matrix composite caused by straining transverse to the fibre direction. Acta Metall Mater 40, 3357-3368.

Wilkinson, A.J., GonZalez, G. \& Dingley, D.J. (1992). The measurement of local plastic deformation in a metal-matrix composite by electron back scatter patterns. J Microsc 169, 255-261.

Wright, S.I. \& Nowell, M.M. (2006). EBSD image quality mapping. Microsc Microanal 12, 72-84. 